\title{
Using of H-Infinity Control Method in Attitude Control System of Rigid-Flexible Satellite
}

\author{
Ximena Celia Méndez Cubillos and Luiz Carlos Gadelha de Souza
}

Space Mechanics and Control Division (DMC), National Institute for Space Research (INPE), Avenida dos Astronautas 1758, P.O. Box 515, São Paulo, 12201-940 São José dos Campos, Brazil

Correspondence should be addressed to Luiz Carlos Gadelha de Souza, gadelha@dem.inpe.br

Received 1 July 2009; Revised 7 October 2009; Accepted 18 November 2009

Recommended by Tadashi Yokoyama

The attitude control systems of satellites with rigid and flexible components are demanding more and more better performance resulting in the development of several methods control. For that reason, control design methods presently available, including parameters and states estimation, robust and adaptive control, as well as linear and nonlinear theory, need more investigation to know their capability and limitations. In this paper the investigated technique is H-Infinity method in the performance of the Attitude Control System of a Rigid-Flexible Satellite.

Copyright (C) 2009 X. C. Méndez Cubillos and L. C. G. de Souza. This is an open access article distributed under the Creative Commons Attribution License, which permits unrestricted use, distribution, and reproduction in any medium, provided the original work is properly cited.

\section{Introduction}

The rapidly complexity increase of systems and processes to be controlled has stimulated the development of sophisticated analysis and design methods called advanced techniques. The H-Infinity $(\mathrm{H} \infty)$ control theory, introduced by Zames [1], is one of the advanced techniques and its application in several problems of control has been growing rapidly.

The employment of flexible structures in the spatial area is another problem of control system which has been growing up too. Flexible systems offer several advantages compared with the rigid system. Some advantages are relatively smaller actuators, lower overall mass, faster response, lower energy consumption, in general, and lower cost. With the study of the Attitude Control System (ACS) of space structures with flexible antennas and/or panel and robotic manipulators, one becomes more complex when the dimensions of such structures increase due to necessity to consider a bigger number of vibration modes in its model in order to improve the model fidelity [2]. Examples of projects that involve flexible space structures are the International Space Station (ISS), the Lunar Reconnaissance Orbiter (LRO), the Lunar Crater Observation and Sensing Satellite (LCROSS), the Hubble Space Telescope, and so forth. 
In Rigid-Flexible Satellite (RFS) the function of the ACS is to stabilize and orient the satellite during its mission, counteracting external disturbances torques and forces. In this paper is investigated multivariable control method Ho for attitude control of an RFS consisting of a rigid body and two flexible panels. The satellite modeling was built following the Lagrangian approach and the discretization was done using the assumed-modes method. The equations of motion obtained were written in its modal state space form.

\section{The Rigid-Flexible Satellite Model}

Figure 1 shows the picture of the satellite used in this work, which is composed of a rigid body of cubic shape and two flexible panels. The center of mass of the satellite is in the point 0 origin of the system of coordinates $(X, Y, Z)$ that coincides with its main axis of inertia. The elastic appendixes with the beam format are connected in the central body, being treated as a punctual mass in its free extremity. The length of the panel is represented by $L$, the mass is represented by $m$, and $v(x, t)$ is the elastic displacement in relation to the axis $Z$. The moment of inertia of the rigid body of the satellite in relation to the mass center is $J_{0}$. The moment of inertia of the panel in relation to its own mass center is given by $J_{p}$.

\section{Equations of Motion}

In the Lagrang approach are considered the equation of motion of the satellite around in $Y$ and the elastic displacement of the panels. The Lagrange equations [3] for the problem can be written in the following form:

$$
\begin{gathered}
\frac{d}{d t}\left(\frac{\partial L^{*}}{\partial \dot{\theta}}\right)-\frac{\partial L^{*}}{\partial \theta}=\tau \\
\frac{d}{d t}\left(\frac{\partial L^{*}}{\partial \dot{q}_{i}}\right)-\frac{\partial L^{*}}{\partial q_{i}}+\frac{\partial M}{\partial q_{i}}=0 .
\end{gathered}
$$

In (3.1) $\tau$ is the torque of the reaction wheel, $L^{*}=T-V$ is the Lagrangian, and $\theta$ is the rotation angle of the satellite around the axis $Y$. In (3.2) $M$ is the dissipation energy associated to the deformation of the panel $q_{i}$ it represents each one of the generalized coordinates of the problem.

The beam deflection variable $v(x, t)$ is discretized using the expansion

$$
v(x, t)=\sum_{i=1}^{n} \phi_{i}(x) q_{i}(t) \quad 0 \leq x \leq L,
$$

where $n$ represents the number of manners to be adopted in the discretization and $\phi_{i}(x)$ represents each one of the own modes of the system. The admissible functions $\phi_{i}(x)$ are given by $[4]$

$$
\phi_{i}(x)=\cosh \left(a_{i} x\right)-\cos \left(a_{i} x\right)-\alpha_{i}\left(\sinh \left(a_{i} x\right)-\sin \left(a_{i} x\right)\right),
$$




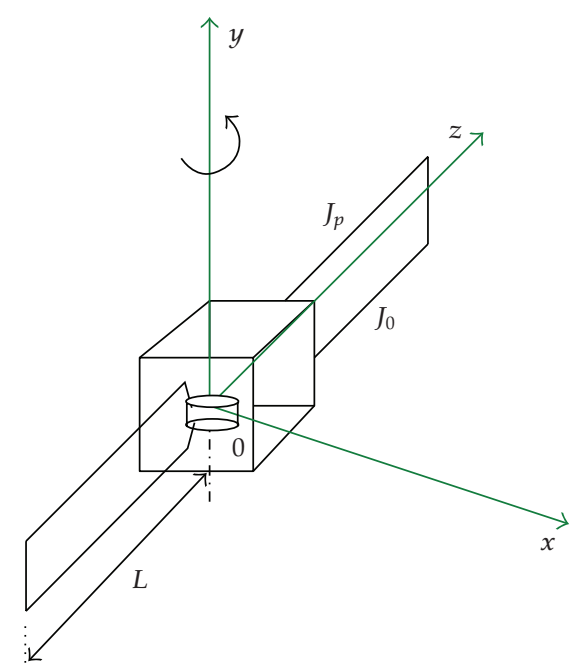

Figure 1: Satellite Model.

where

$$
\alpha_{i}=\frac{\cosh \left(a_{i} L\right)+\cos \left(a_{i} L\right)}{\sinh \left(a_{i} L\right)+\sin \left(a_{i} L\right)}
$$

and $a_{i} L$ are the eigenvalues of the free system and undamped.

For the complete system, the total kinetic energy $T$ is given by $T=T_{\text {Satellite }}+T_{\text {Panel }}$; therefore,

$$
T=\frac{1}{2} J_{0} \dot{\theta}^{2}+\left[\rho A^{\prime} \int_{0}^{L}\left[\dot{v}(x, t)^{2}+2(\dot{v}(x, t)) x \dot{\theta}+(x \dot{\theta})^{2}+(\dot{\theta} \cdot \dot{v}(x, t))^{2}\right] d x\right],
$$

where $\rho$ is the density of the panels and $A^{\prime}$ is the area. The dissipation energy function is

$$
M=\dot{v}(x, t)^{2} K_{d}
$$

where $K_{d}$ is the dissipation constant. So $L^{*}=T-V$ is given by

$$
L^{*}=\frac{1}{2} J_{0} \dot{\theta}^{2}+\left[\rho A^{\prime} \int_{0}^{L}\left[\dot{v}(x, t)^{2}+2(\dot{v}(x, t)) x \dot{\theta}+(x \dot{\theta})^{2}+(\dot{\theta} \cdot \dot{v}(x, t))^{2}\right] d x\right]-v(x, t)^{2} \cdot K .
$$

In (3.8) $\mathrm{K}$ is constant elastic of the panels. After some manipulations of (3.8) [5] and using the orthogonalization property of vibration modes of the beam [6], one has

$$
\int_{0}^{L} \phi_{i} \phi_{j} d x=1 \quad \text { if } i=j, \quad \int_{0}^{L} \phi_{i} \phi_{j} d x=0 \quad \text { if } i \neq j .
$$


Finally, two equations are obtained. These equations represent the dynamics of rotation motion of the satellite and the elastic displacement of the panels, respectively,

$$
\begin{gathered}
\ddot{\theta}\left(1+a \sum_{i=1}^{n} q_{i}^{2}\right)+\alpha_{i} \cdot a \sum_{i=1}^{n} \ddot{q}_{i}=\frac{1}{J_{1}} \tau . \\
\ddot{q}_{i}+\alpha_{i} \ddot{\theta}-\dot{\theta}^{2} q_{i}+d \cdot \dot{q}_{i}+c \cdot q_{i}=\tau_{q} .
\end{gathered}
$$

where the term nonlinear $\alpha_{i}$ in (3.10) is defined as centripetal rigidity, and in (3.11) $\tau_{q}$ is a Piezoelectric actuator adapted for the following simulations where will be considered $i=1$ (one mode), and the constants are given by

$$
a=\frac{2 \rho A^{\prime}}{J_{1}}, \quad J_{1}=J_{0}+2 J_{p}, \quad c=\frac{K}{\rho A^{\prime}}, \quad d=\frac{K_{d}}{\rho A^{\prime}} .
$$

\section{H-Infinity Control Method}

\subsection{Introduction}

Throughout the decades of 1980 and 1990, H-Infinity control method had a significant impact in the development of control systems; nowadays the technique has become fully grown and it is applied on industrial problems [5]. In the control theory in order to achieve robust performance or stabilization, the H-Infinity control method is used. The control designer expresses the control problem as a mathematical optimization problem finding the controller solution. Hoo techniques have the advantage over classical control techniques in which the techniques are readily applicable to problems involving multivariable systems with cross-coupling between channels; disadvantages of $\mathrm{H} \infty$ techniques include the high level of mathematical understanding needed to apply them successfully and the need for a reasonably good model of the system to be controlled. The problem formulation is important, since any synthesized controller will be "optimal" in the formulated sense.

The $\mathrm{H} \infty$ name derives from the fact that mathematically the problem may be set in the space $\mathrm{H} \infty$, which consists of all bounded functions that are analytic in the right-half complex plane. We do not go to this length. The Hoo norm is the maximum singular value of the function; let us say that it can be interpreted as a maximum gain in any direction and at any frequency; for SISO (Single In, Single Out) systems, this is effectively the maximum magnitude of the frequency response. Ho method is also used to minimize the closedloop impact of a perturbation: depending on the problem formulation, the impact will be measured in terms of either stabilization or performance. Thus, one concludes that the procedures to project control systems are a difficult task due to the cited terms which are conflicting properties [7].

\subsection{Modeling}

This problem is defined by the configuration of Figure 2. The "plant" is a given system with two inputs and two outputs. It is often referred to as the generalized plant. The signal $w$ is an external input and represents driving signals that generate disturbances, measurement noise, and reference inputs. The signal $u$ is the control input. The output $z$ has the meaning 


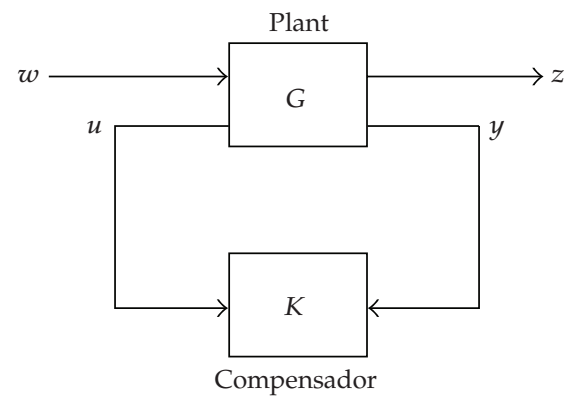

Figure 2: Generalized Plant.

of control error and ideally should be zero. The output $y$, finally, is the observed output and is available for feedback.

The project of control system is based given by

$$
\begin{aligned}
& \dot{x}(t)=A x+B u, \\
& y(t)=C x+D u .
\end{aligned}
$$

A more general state space representation of the standard plant is

$$
\begin{aligned}
\dot{x}(t) & =A x(t)+B_{1} w(t)+B_{2} W(t), \\
z(t) & =C_{1} x(t)+D_{11} w(t)+D_{12} u(t), \\
y(t) & =C_{2} x(t)+D_{21} w(t)+D_{22} u(t), \\
{\left[\begin{array}{l}
\dot{x}(t) \\
z(t) \\
y(t)
\end{array}\right] } & =\left[\begin{array}{lll}
A & B_{1} & B_{2} \\
C_{1} & D_{11} & D_{12} \\
C_{2} & D_{21} & D_{22}
\end{array}\right] \cdot\left[\begin{array}{l}
x(t) \\
w(t) \\
u(t)
\end{array}\right]=P .
\end{aligned}
$$

The solution of the corresponding Hoo problem based on Riccati equations is implemented requires the following conditions to be satisfied [8]:

(1) $\left(A, B_{2}\right)$ is stabilizable and $\left(C_{2}, A\right)$ is detectable,

(2) $D_{12}$ and $D_{21}$ have full rank,

(3) $\left[A-j w I B_{2} ; C_{1} D_{12}\right]$ has full column rank for all $w \in \mathfrak{R}$ (hence, $D_{12}$ is tall),

(4) $\left[A\right.$-jwI $\left.B_{1} ; C_{2} D_{21}\right]$ has full column rank for all $w \in \mathfrak{R}$ (hence, $D_{21}$ is wide).

The augmented plant is formed by accounting for the weighting functions $W_{1}, W_{2}$, and $W_{3}$, as shown in Figure 3. In order to reach the acting objectives, the outputs were chosen to be transfer weight functions: $z_{1}=W_{1} ; z_{2}=W_{2} y ; z_{3}=W_{3} u$. 


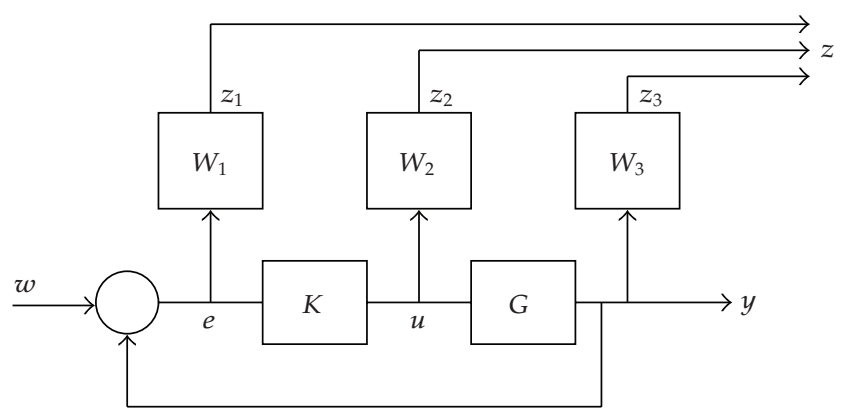

Figure 3: Plant with weighting functions for Hळ design.

Table 1: Parameters.

\begin{tabular}{lcc}
\hline Parameter & Description & Value \\
\hline$J_{0}$ & Moments of inertia of the rigid body of the satellite & $720 \mathrm{Kg} \cdot \mathrm{m}^{2}$ \\
$J_{p}$ & Moment of inertia of the panel & $40 \mathrm{Kg} \cdot \mathrm{m}^{2}$ \\
$K$ & Constant elastic of the panels & $320 \mathrm{Kg} \cdot \mathrm{rad}^{2} / \mathrm{s}^{2}$ \\
$K_{d}$ & Dissipation constant & $0,48 \mathrm{Kg} \cdot \mathrm{rad}^{2} / \mathrm{s}$ \\
$L$ & Length of the panel & $2 \mathrm{~m}$ \\
$m$ & Mass of the satellite & $200 \mathrm{~kg}$ \\
\hline
\end{tabular}

The function cost of mixed sensibility is given for

$$
T_{y 1 u 1}=\left[\begin{array}{c}
W_{1} S \\
W_{2} R \\
W_{3} T
\end{array}\right], \quad \begin{gathered}
S=(I+G K)^{-1}, \\
T=G K(I+G K)^{-1},
\end{gathered}
$$

where $S$ is called sensibility, $T$ is complementary sensitivity function, and $R$ does not have any name. The cost function of mixed sensibility is named alike, because it punishes $S, R$, and $T$ at the same time; it can also be said project requirement. The transfer function from $w$ to $z_{1}$ is the weighted sensitivity function $W_{1} S$, which characterizes the performance objective of good tracking; the transfer function from $w$ to $z_{2}$ is the complementary sensitivity function $T$, whose minimization ensures low control gains at high frequencies, and the transfer function from $w$ to $z_{3}$ is $K S$, which measures the control effort. It is also used to impose the constraints on the control input for example, the saturation limits.

\section{Simulations}

The simulations were carried out by computational implementation of the software MatLab. The initials conditions used here are $\theta=0.001 \mathrm{rad}$. and $\dot{\theta}=0 \mathrm{rad}$./s. The values considered for the physical parameters in the numerical simulation are presented in Table 1.

The procedure of the project of $\mathrm{H} \infty$ is different from other control projects knowledge such as LQR (Linear Quadratic Regulator) and LQG (Linear Quadratic Gaussian) [5]; the 


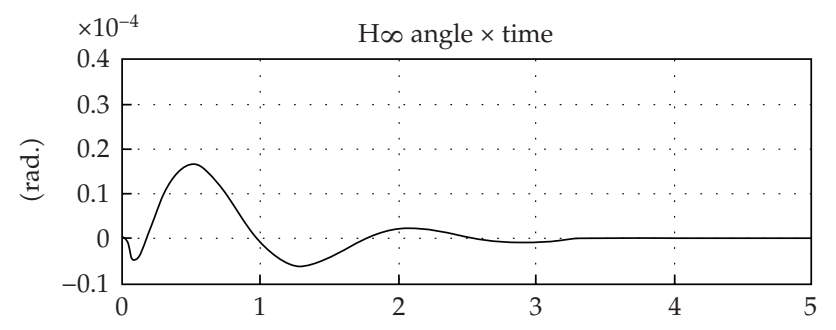

(s)

(a)

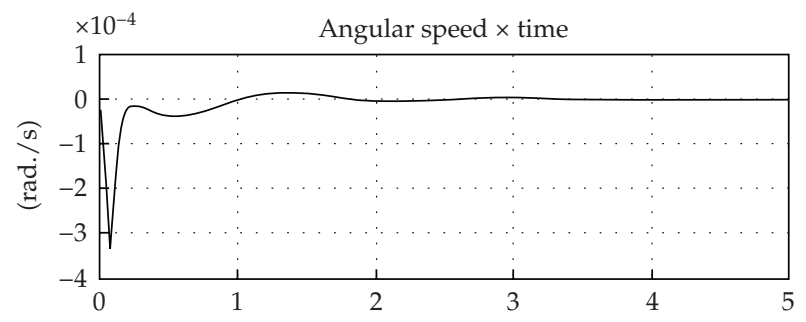

(s)

(b)

Figure 4: Angle and Angular Speed.

difference is the use of weighting functions $W_{1}, W_{2}$, and $W_{3}$, where $W_{2}=0$ and the others are given by

$$
\begin{aligned}
& W_{1}^{-1}=r^{-1} * \frac{0.1(1+s / 100)^{2}}{(1+s / 5000)^{2}} * I_{2 \times 2}, \\
& W_{3}^{-1}=\frac{2000}{s} * I_{2 \times 2},
\end{aligned}
$$

where $W_{1}$ punishes the error sign $e, W_{2}$ punish the control sign " $u$ ", and $W_{3}$ punishes the exit of the plant $y ; \gamma$ is a parameter obtained through successive attempts.

\section{Results}

First we analyze the open loop of the system through transmission zeros (TZs) and the closeloop with Ho Control. The TZs are critical frequencies where signal transmission between input and output is stopped. The importance of use of the TZs is given by their application in robust control, because they are the zeros of a MIMO system. In Table 2 are represented the values.

Following, the performances of Hoo control in the ACS are observed in Figures 4 and 5.

Both graphs, in Figure 4, have existence of overshoot, in which they could commit the system; however, the time of stabilization of both was of approximately 3.5 seconds. In other words, in spite of the existence of overshoots, the control of the system, in a long time, was reached. 


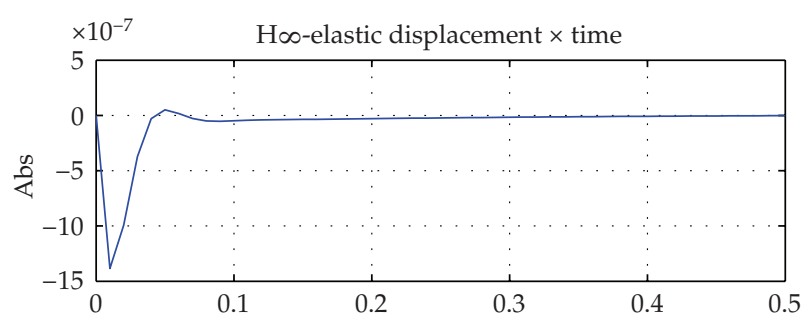

(s)

(a)

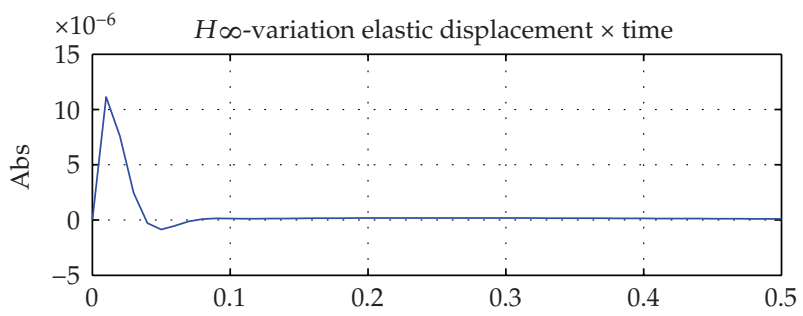

(s)

(b)

Figure 5: Vibration of the Panels.

Table 2: Transmission Zeros.

\begin{tabular}{lc}
\hline & Transmission Zeros \\
\hline Open Loop & $-0.0244 \pm 5.7049 i$ \\
\hline & -2318.10 \\
Ho Control & -433.3 \\
& $-0.0 \pm 05.700 i$ \\
& $-0 \pm 0 i$ \\
\hline
\end{tabular}

In Figure 5 the behavior of the vibration of the panels is presented. The displacement of overshoot is of the order of $10^{-7}$, in other words, very small. The time of stabilization in the first graph is about 0.5 seconds and for the second one is about 0.45 seconds. This demonstrates that the control Hoo possesses a good performance for angle and angular velocity, as well as to control the vibration of the panels.

\section{Conclusions}

The problem of attitude control of satellites is not new and has been addressed by several researchers using many different approaches. The Hos control method is one of the most advanced techniques available today for designing robust controllers. One great advantage with this technique is that it allows the designer to tackle the most general form of control architecture wherein explicit accounting of uncertainties, disturbances, actuator/sensor noises, actuator constraints, and performance measures can be accomplished. The system is very different from the methods LQR and LQG, for example. However, a great disadvantage is the experience and necessary abilities to design the form of the weighting functions and 
the fact that the plant can increase. Basically, the success of the method depends on the correct choice of the weight functions transfer.

\section{Acknowledgments}

The authors would like to thank CAPES and INPE/DMC. This work was supported by CAPES thought the Brasil-Portugal Cooperation Project PCT no. 241/09.

\section{References}

[1] G. Zames, "Feedback and optimal sensitivity: model reference transformations, multiplicative seminorms, and approximate inverses," IEEE Transactions on Automatic Control, vol. 26, no. 2, pp. 301320, 1981.

[2] L. C. G. De Souza and S. A. Silva, "Vibration control of a rigid-flexible satellite during attitude maneuver," in Proceeding of the 17th ASME Biennial Conference on Mechanical Vibration and Noise (DETC'99), Las Vegas, Nev, USA, September 1999.

[3] L. Meirovitch, Methods of Analytical Dynamics, Dover, Mineola, NY, USA, 1998.

[4] J. L. Junkins and Y. Kim, Introduction to Dynamics and Control of Flexible Structures, AIAA, Washington, DC, USA, 1993.

[5] X. C. M. Cubillos, Investigation of Multivariable Control Techniques for Attitude Control of a Rigid-Flexible Satellite, INPE, São José dos Campos, Brazil, 2008.

[6] C. H. G. Hassmann, and A. Fenili, "Attitude and vibration control of a satellite with a flexible solar panel using Lqr tracking with infinite time," in Proceedings of the ASME International Congress of Mechanical Engineering, Seattle, Wash, USA, November 2007.

[7] M. G. Safonov, A. J. Laub, and G. L. Hartmann, "Feedback properties of multivariable systems: the role and use of the return difference matrix," IEEE Transactions on Automatic Control, vol. 26, no. 1, pp. 47-65, 1981.

[8] O. H. Bosgra and H. Kwakernaak, "Design methods for control systems," Notes for a Course of the Dutch Institute of Systems and Control, Winter term 2001-2002. 


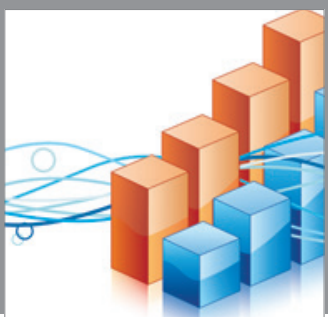

Advances in

Operations Research

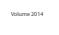

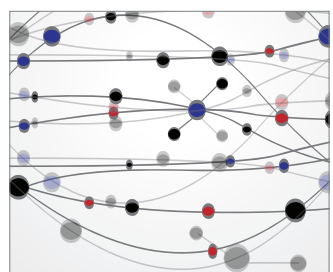

\section{The Scientific} World Journal
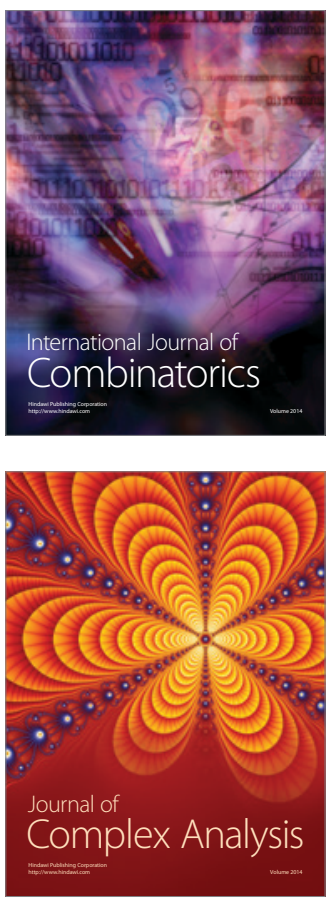

International Journal of

Mathematics and

Mathematical

Sciences
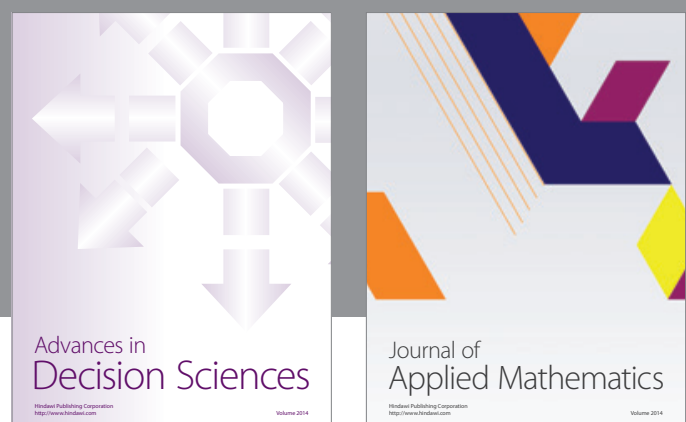

Journal of

Applied Mathematics
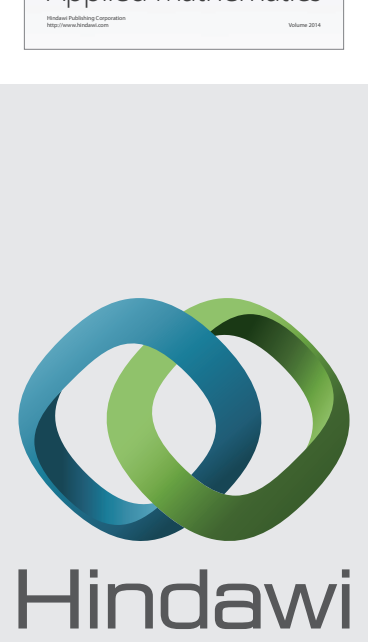

Submit your manuscripts at http://www.hindawi.com
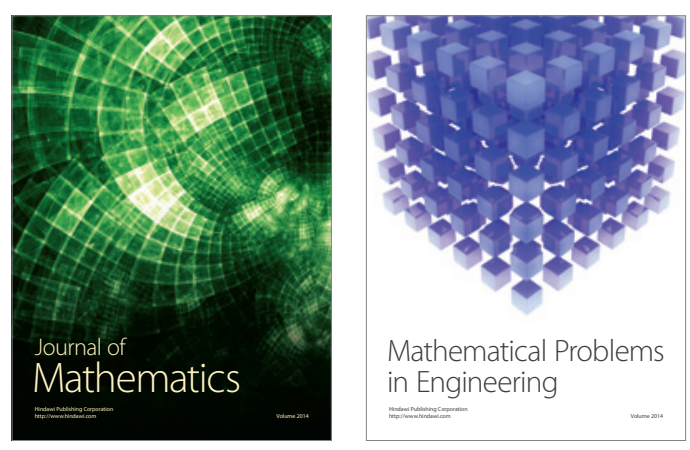

Mathematical Problems in Engineering
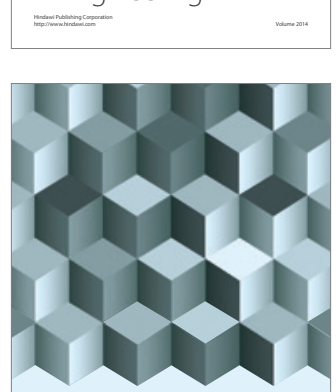

Journal of

Function Spaces
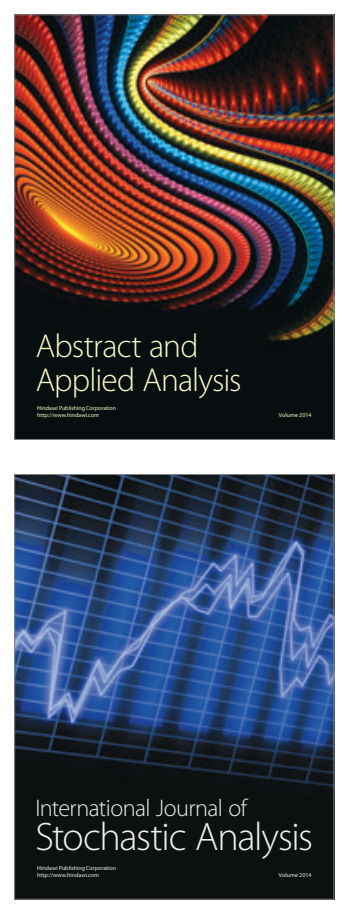

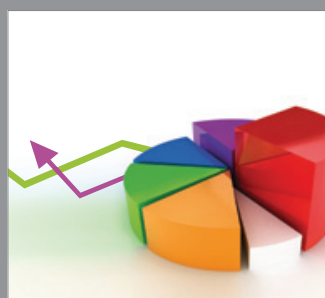

ournal of

Probability and Statistics

Promensencen
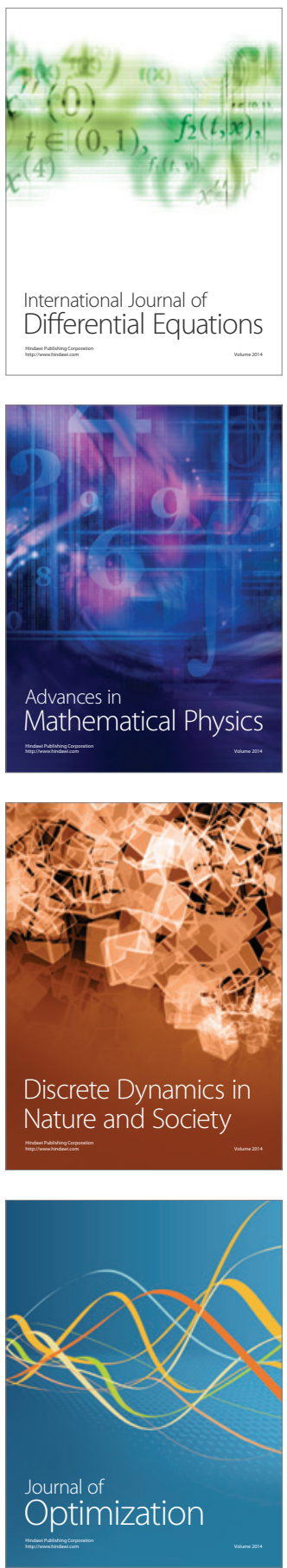